complicates, deformities can be the more easily induced. We would not resort to massage or electricity or to vibromassage or to any of those excitants of the muscles or nerves until we feel pretty sure that all inflammatory changes have undergone resolution in the cerebrospinal axis.

We would like to have the counsel then of a neurologist who would conduct the electric treatment. For it must be understood now that orthopedic surgeons do not as a rule discourage these valuable aids in cases of this kind. We do sometimes object to the use of electricity in the paralyses of many years' standing, but during the first year we feel that every aid must be employed to bring about the best possible result. We do object, however, to fatigue of the muscles or strain by having the children urged to swing about a dangleleg and bear weight on a weakened joint. The do object to having these partially paralyzed muscles strained berond endurance. and we do believe that, if such measures are emploved, further destruction of the ganglion cells in the anterior cornua is a result. In other words, we believe in husbanding all our resources and in this way encouraging regenerations of these ganglion cells, and we use this term with all due respect to the teachings of pathology.

We aim, furthermore, to avoid straining the capsule or ligaments of the joint, because when these are weakened the muscles are called on to still greater efforts. We believe in preventing deformity rather than having to correct it. We believe, finally, that if the limbs are kept in normal position, all these therapeutic aids which go to the overdeveloping of the muscle fibers not completely destrored, to the stimulation of weakened muscle fibers in danger of acute atrophy, and to improving the circulation of the limb (terms mighty popular with the advocates of all forms of elcetricity, massage, vacuum treatment, et id omne genus) - all will be much more efficient, and our prognoses will be infinitely better than they have been in the past.

We do object to interference on the part of well-meining friends in and out of the profession. Such interference as this: "Take off that heavy brace and let the child use the limb and make him walk." Many and many a time patients have followed such advice and have come back to the same form of apparatus and have derived benefit even when applied in the later stages of the disease. We would adrocate, as a further remedial measure in these early cases, artificial hyperemia of the parts in the neighborhood of the cord, such as cupping in the younger children, the Paquelin cautery in the older ones, iodin, and other forms of counter-irritation, if more vigorous ones can not be employed. If there are intestinal complications let the family physician help out in taking care of this factor.

We have been able to treat six or seven cases in the hospital over a period of from six to eight weeks, and practically no treatment has been employed other than rest in bed. In looking over the notes of these cases we find that none have been under observation sufficiently long to be utilized in this communication.

In discussing certain features of the epidemic with colleagues we are impressed with the pain element which characterizes a majority of the cases that have come under observation at the Hospital for Ruptured and Crippled. One recent writer in Pediatrics, Dr. Pisek, speaks of the involvement of the extensor spinæ group. The case of one of our patients now in the hospital will confirm this statement:
History.-A boy, aged 5, was admitted to the hospital October 7,1907 , with this history: Four weeks prior to this date fever developed, headache, anorexia, aches and pains in left upper extremity and the next day the shoulder group was - paralyzed. He remained in bed for two weeks very ill, and the shoulder group and erector spinæ muscles were still paralyzed. Examination showed that he had a very unsteady gait, was unable to elevate the shoulders, and we were struck with his total inability to turn over in bed or to assume the sitting posture. The erector spinæ muscles, therefore, were involved as well as those of the shoulder group. At the date of this writing, November 7 , he is able to sit up in bed easily, and there has been decided improvement in the shoulder group of muscles.

In studying the reports of former epidemics we fail to find anything quite as complete in a pathologic way as is presented in a paper of Harbitz and Scheel. to which reference has already been made. These observers base their conclusions on the study of 19 fatal cases, in every one of which an autopsv was made. The time intervening between the invasion and death was as follows: In 1 case, 2 dars; in 1,3 days; in 1 , 4 days; in 1,5 dars; in 8 , from 7 to 9 days, and in 1 , 10 days.

There were four cases of acute poliomyelitis where death occurred during the stage of repair, namely, 1.5, 6.5, 6.5 and 22.5 months after the onset. The details of the autopsies are extremely interesting, and it is difficult to quote from this raluable paper without incorporating the major portion of it, but their conclusion is worthy of record here, and it is this:

It is our opinion that poliomyelitis is due to a specific virus, and, further, that we are not dealing with the remote effect on the central nervous system, but that the organism is present in the organism itself, in the meninges, in the cerebrospinal fluid and probably in the nervous substance, and that it is the direct cause of the extensive inflammation. As has been stated, many facts favor the assumption that the atrium of the infection is in the digestive tract and that the nervous system becomes infected either by the lymph stream, along vessels and nerve trunks (though no anatomic proof of this exists) or, what appears more likely to us, by way of the blood, which also appears to be probably the case in cerebrospinal meningitis.

\section{Clinical Notes}

\section{IMPROVED CARBOLIC FUCHSIN STAIN FOR TUBERCLE BACIJLUS.}

\author{
R. O. LeBARON, B. Sc., M.D. \\ PORTSMOLTH, OHIO.
}

The staining solution is made by saturating methyl alcohol (9\% per cent.) with fuchsin and adding 5 per cent. of carbolic acid. This fluid will stain the tubercle bacilli in sputum in four minutes without employing heat. As the stain is dissolved in methyl alcohol, preliminary fixation is really unnecessary, but I have found that, at least with some sputums, a smoother field is obtained by a light fixation in the flame. (The sputums were all incubated for from twelve to twenty-four hours.) The specimen is then covered with the stain, which is allowed to remain at least four, but not more that five, minutes, timed by the watch; washed thoroughly in water; decolorized (dilute nitric acid and 60 per cent. alcohol used separately), and counterstained as ordinarily. The counterstain should be rather light. The tubercle bacilli are stained deeply and the field is unusually clear. 